\title{
CAUSES FOR READMISSIONS IN PATIENTS WITH MULTIDRUG RESISTANT TUBERCULOSIS IN A TERTIARY CARE CENTRE
}

\author{
K. Rajarajan', N. Sukanya², G. Allwyn Vijay3
}

${ }^{1}$ Assistant Professor, Government Dharmapuri Medical College, Dharmapuri, Tamilnadu.

${ }^{2}$ Assistant Professor, Government Stanley Medical College, Chennai, Tamilnadu.

${ }^{3}$ Associate Professor, Department of Thoracic Medicine, Government Villupuram Medical College, Villupuram, Tamilnadu.

\begin{abstract}
\section{BACKGROUND}

Multidrug Resistance Tuberculosis has emerged as a significant public health problem and an obstacle for effective control of Tuberculosis. ${ }^{1}$ MDR-TB, by nature of the disease and long duration of the treatment has significant impact on health and socioeconomic aspects of individual and society. ${ }^{2}$

Objective-The objective of the study was to assess the main causes for readmissions at GHTM, Tambaram, in MDR TB patients during treatment, identifying the main variables which lead to a longer hospitalisation time.
\end{abstract}

\section{MATERIALS AND METHODS}

Study Period: July 2009 to January 2013.

Study Design: Retrospective study. Data is obtained from the DR-TB register and THIS (Tuberculosis and HIV Information system) available at our Institute. The In-Patient records are analysed and further details of admissions are obtained.

\section{Inclusion Criteria}

All patients diagnosed as MDR TB were started on treatment and having readmitted are included in the study.

Exclusion Criteria

Patients readmitted after completion of MDR regimen are excluded from the study.

\section{RESULTS}

Total of 790 patients were analysed, out of which 414 (52.4\%) were readmitted. Mean duration of symptoms prior to admission is 34.6 days. Mean duration of stay in Institution is 19.8 days.

\section{CONCLUSION}

Adverse drug reaction (34.7\%), Default (12.5\%) and Complications of MDR TB (16.6\%) are common causes for readmission. Social causes have significant proportion of readmission (9\%). Readmissions are more common in patients with HIV and substance abuse.

\section{KEYWORDS}

MDR TB, Readmissions.

HOW TO CITE THIS ARTICLE: Rajarajan K, Sukanya N, Vijay GA. Causes for readmissions in patients with multidrug resistant tuberculosis in a tertiary care centre. J. Evolution Med. Dent. Sci. 2017;6(35):2898-2902, DOI: 10.14260/Jemds/2017/625

\section{BACKGROUND}

Multidrug Resistance Tuberculosis has emerged as a significant public health problem and an obstacle for effective control of Tuberculosis. ${ }^{1}$ MDR-TB, by nature of the disease and long duration of the treatment has significant impact on health and socioeconomic aspects of individual and society. ${ }^{2}$ Patients are probably admitted in Tertiary Care Institutes due to low access to standard healthcare facility to the patient, low resolving capacity of the health teams in nearby areas, severe cases with long lasting symptoms, ${ }^{3}$ more in patient admission adds up to the cost of treating MDR TB. ${ }^{4}$

Financial or Other, Competing Interest: None.

Submission 18-02-2017, Peer Review 16-04-2017,

Acceptance 22-04-2017, Published 01-05-2017.

Corresponding Author:

Dr. G. Allwyn Vijay,

Department of Thoracic Medicine,

Government Villupuram Medical College,

Mundiyampakkam, Villupuram-605601,

Tamilnadu.

E-mail: allwynvijay28@gmail.com

DOI: $10.14260 /$ jemds $/ 2017 / 625$

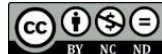

Worse than this MDR TB patients not only need admissions in the initial diagnosis and initiation of treatment, they need multiple readmissions in the course of treatment that comes to 24 months. It is a high burden situation for the individual, the family and for the state economically as well as emotionally.

Shradha Bhagawat et al ${ }^{5}$ did a similar study in Maharashtra, India. The aim of this study is to identify causes of hospitalisation in tuberculosis and to assess clinical profile and outcome of patients hospitalised for tuberculosis. This study saw that Tuberculosis is a disease having significant impact on the patient and the society as a whole. Hospital admissions for tuberculosis can occur due to various reasons such as haemodynamic instability, hypoxia, severe forms of tuberculosis, adverse drug reactions to anti-tuberculosis drugs for the purpose of certain investigations and also for various socioeconomic reasons such as homelessness, insufficient social support, etc.

There is another similar study done in Brazil, which shows similar facts in drug sensitive $\mathrm{Tb}$ patients...J. Pneumologia Vol. 29 No. 1 São Paulo Jan./Feb. 2003. The duration of symptoms before hospital admission was 2.9 months on the average, a period similar to the one observed 
in the basic healthcare network until a diagnosis is made (approximately three months). This is probably due to the worse general conditions presented by patients with a severe illness, justifying hospitalisation. Thus, malnourished, alcoholics, HIV-positive patients, individuals under stress and in poor socioeconomic situation (regarding home, food, etc.) are more susceptible to presenting severe forms of $\mathrm{Tb}$ including bronchogenic or lymphohematogenic dissemination, leading them to go to a general hospital as a first step and to be admitted there. Therefore, a good tuberculosis control program should improve the access of patients at high risk for $\mathrm{Tb}$ to the healthcare services, in order to prevent the spreading of the disease among them and the persons they are in contact with and active search for new $\mathrm{Tb}$ cases should be present in any healthcare program for early diagnosis of the disease.

This study at Government Hospital of Thoracic Medicine, Tambaram is done with the intention that there could be a possible identification of factors that can be possibly averted. So there is an assessment of various factors relating to readmissions of MDR TB patients in this study.

Government Hospital of Thoracic Medicine, Tambaram is a Tertiary Care Institute Pioneers in Tb Research Treatment and Care. It is also a centre of excellence for HIV related disorders.

\section{Objective}

The objective of the study was to assess the main causes for readmissions at GHTM, Tambaram in MDR TB patients during treatment, identifying the main variables which lead to a longer hospitalisation time.

\section{MATERIALS AND METHODS}

Government Hospital of Thoracic Medicine (GHTM), Tambaram is a Tertiary Care Institution for Thoracic Medicine.

\section{Study Period}

July 2009 to January 2013.

\section{Study Design}

Retrospective study. Data is obtained from the DR-TB register and THIS (Tuberculosis and HIV Information System) available at our Institute. The In-Patient records are analysed and further details of admissions are obtained. This study analyses the causes of readmissions in our Institution (790 patients with MDR TB and 419 readmitted MDR TB patients), Comorbidities, number of readmissions of each patient, duration of admission, Male:Female ratio, HIV status, substance abuse. In all these cases, MDR TB was diagnosed by line probe assay method and conventional LJ medium solid culture.

\section{Inclusion Criteria}

All patients diagnosed as MDR TB started on treatment and having readmitted are included in the study.

\section{Exclusion Criteria}

Patients readmitted after completion of MDR regimen are excluded from the study.

\section{RESULTS}

Total of 790 patients were analysed, out of which 414 $(52.4 \%)$ were readmitted. Mean duration of symptoms prior to admission is 34.6 days. Mean duration of stay in Institution is 19.8 days.

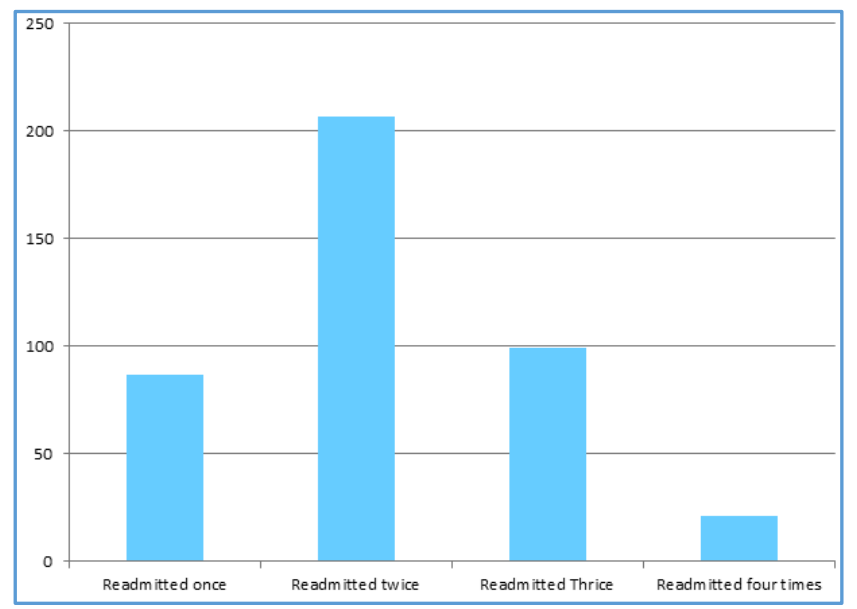

\section{Number of Readmissions}

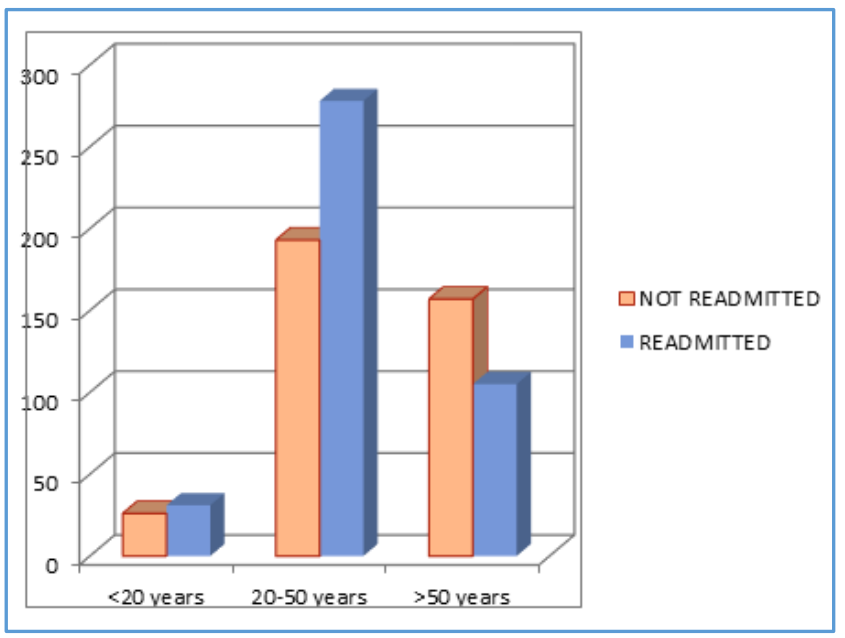

Age Group

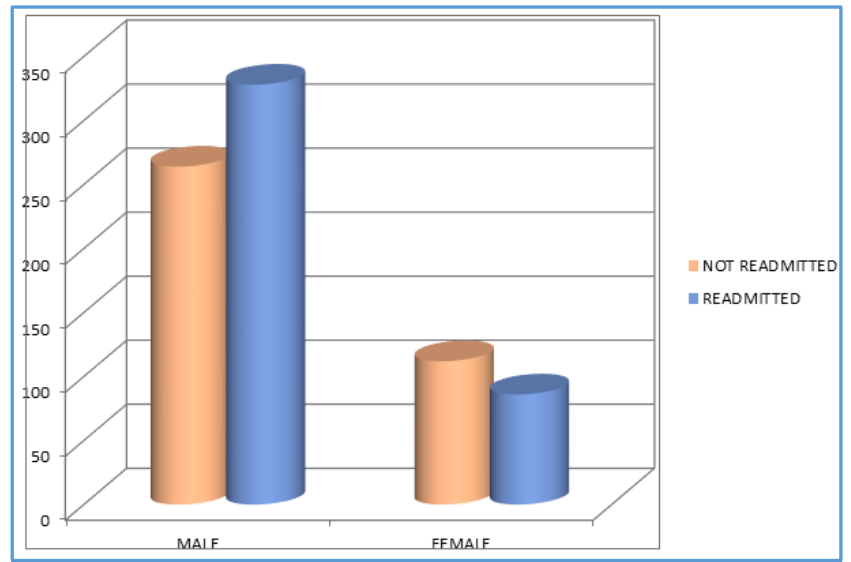




\section{Sex Distribution}

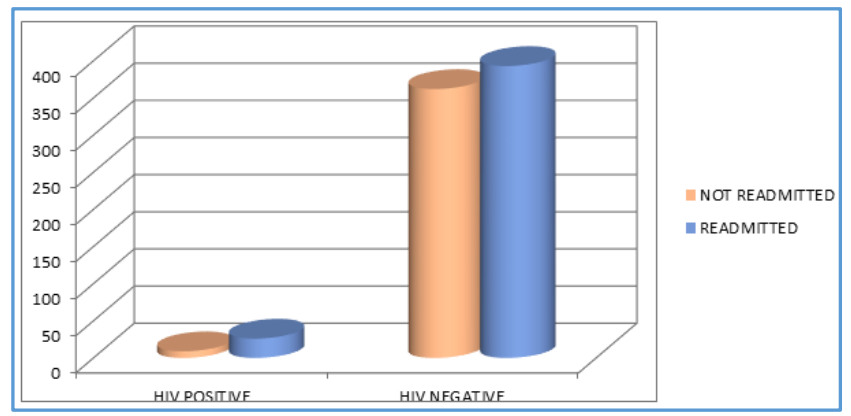

HIV positive patients have significantly higher number of readmissions (75\%) compared to HIV negative patients.
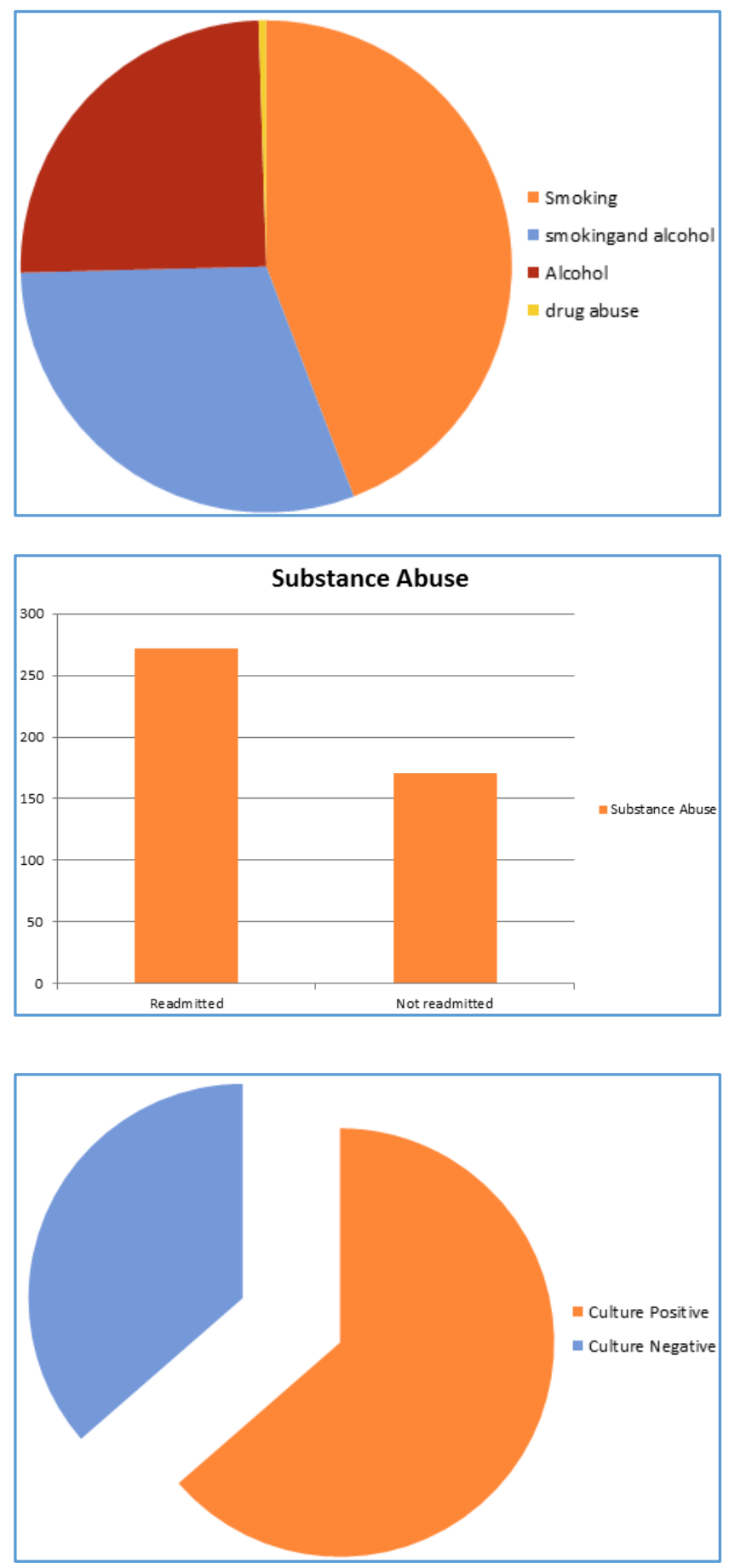

\section{Mycobacterial Culture Status}

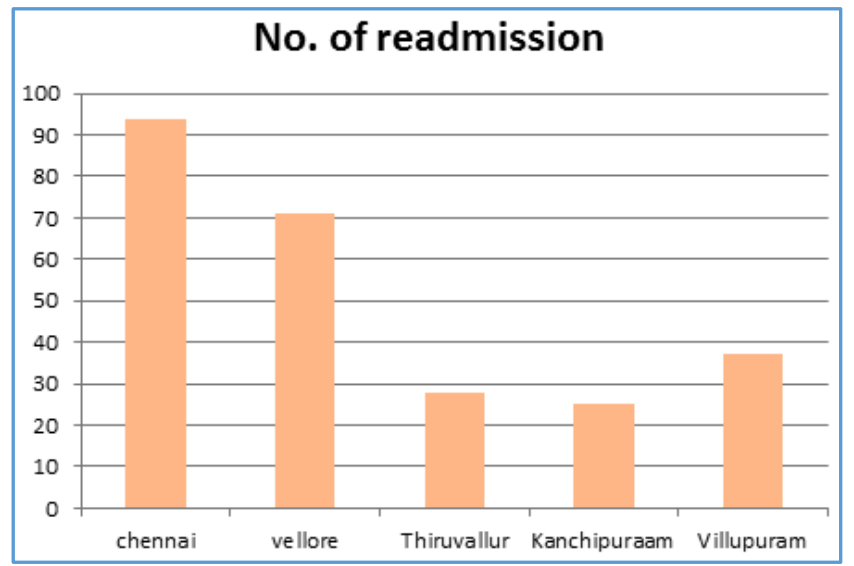

Distance from the Admitting Centre

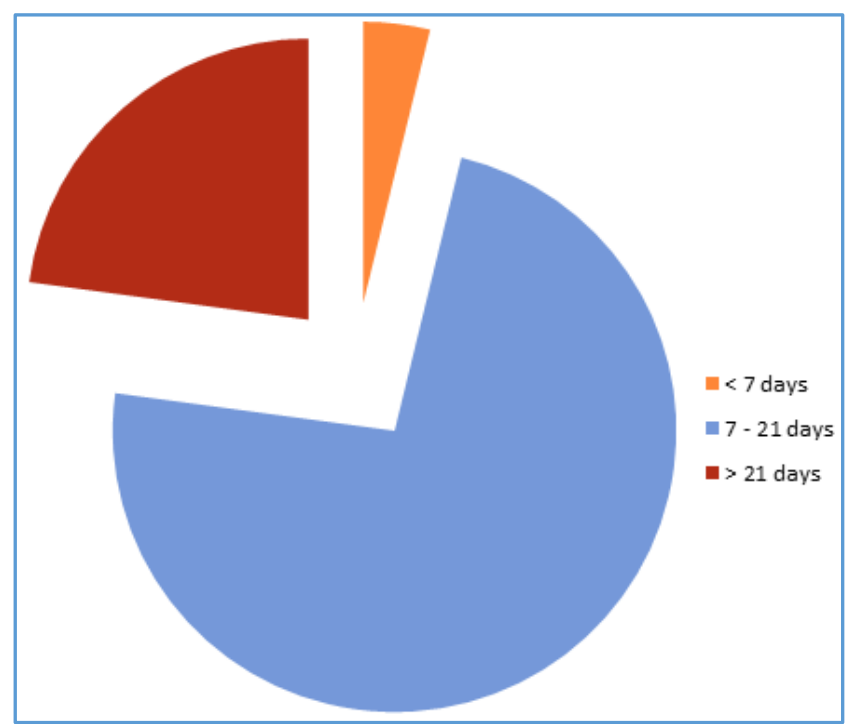

Average Duration of Stay

\begin{tabular}{|l|c|}
\hline Respiratory Distress & $82(19.8 \%)$ \\
\hline Defaulter & $52(12.6 \%)$ \\
\hline Adverse Drug Reactions & $144(34.7 \%)$ \\
\hline Social Causes & $27(6.5 \%)$ \\
\hline Complications of MDR TB & $65(15.7 \%)$ \\
-Haemoptysis & $32(7.7 \%)$ \\
-Pneumothorax, Hydropneumothorax & $14(3.3 \%)$ \\
-BPF & $11(2.6 \%)$ \\
-Disseminated Tb & $8(1.9 \%)$ \\
\hline CCF/Cor pulmonale & $18(4.3 \%)$ \\
\hline Renal Failure & $8(1.9 \%)$ \\
\hline Decompensated Liver Disease & $5(1.2 \%)$ \\
\hline Others & $13(3.1 \%)$ \\
\hline
\end{tabular}

\begin{tabular}{|c|c|}
\hline Exacerbation of Respiratory Symptoms & $197(47.5 \%)$ \\
\hline Haemoptysis & $32(7.7 \%)$ \\
\hline Chest Pain & $15(3.6 \%)$ \\
\hline Fever & $13(3.1 \%)$ \\
\hline Extra Pulmonary Symptoms & \\
\hline
\end{tabular}




\begin{tabular}{|c|c|}
\hline $\begin{array}{c}\text { Abdominal Pain, Nausea, Diarrhoea, } \\
\text { Vomiting, Jaundice }\end{array}$ & $58(14.31 \%)$ \\
\hline Arthralgia & $23(5.55 \%)$ \\
\hline Skin Rash & $21(5.31 \%)$ \\
\hline Seizures, Headache, Tingling, Numbness & $20(4.83 \%)$ \\
\hline Vertigo, Tinnitus, Hard of Hearing & $13(3.1 \%)$ \\
\hline Psychiatric Illness & $8(1.9 \%)$ \\
\hline Others & $13(3.1 \%)$ \\
\hline
\end{tabular}

\section{Adverse Effects}

\begin{tabular}{|c|c|}
\hline GI Intolerance & $41(28.4 \%)$ \\
\hline Arthralgia & $23(15.8 \%$ \\
\hline Skin Rash & $21(14.6 \%)$ \\
\hline Hepatitis & $20(14.3 \%)$ \\
\hline Peripheral Neuropathy & $14(9.7 \%)$ \\
\hline Kanamycin induced Ototoxicity & $13(9 \%)$ \\
\hline Cycloserine-induced Psychosis & $8(5.5 \%)$ \\
\hline Others & $4(2.7 \%)$ \\
\hline
\end{tabular}

\begin{tabular}{|c|c|}
\hline Anaemia & $95(23.4 \%)$ \\
\hline Diabetes & $134(32.4 \%)$ \\
\hline SHT & $31(7.4 \%)$ \\
\hline HIV & $25(6.07 \%)$ \\
\hline CAD & $21(5.1 \%)$ \\
\hline
\end{tabular}

\section{Comorbidities}

Total number of patients admitted under MDR TB ward during the assessment period is 790 and those who were readmitted were 419 , which are approximately more than $50 \%$. Mean duration of stay in these patients is about 20 days. Patients were readmitted once, twice or sometimes four times in the study period. Significant proportions were admitted twice.

Readmissions were maximum in the age group of $20-50$ compared to those in the age group of $<20$ and $>50$ years. This results in significant loss of work hours due to repeated hospitalisations.

Male patients were more commonly admitted than the female. Significant percentage of HIV positive individuals had readmissions comparing to those who were HIV negative.

Significant number of patients had alcohol and substance abuse and it was interesting to see more number of readmissions in the abuse group.

Culture conversion was another significant factor in the readmission causes. Early culture converters got less readmission numbers than those who were non-culture converters. Early identification of disease and early initiation of treatment and thereby early culture conversion will lead to lesser readmissions.

Distance of the residence of MDR TB patients did not have significant impact on the readmissions.

Vellore which is about $170 \mathrm{kms}$ from the Government Hospital of Thoracic Medicine, Tambaram had more admissions than much more nearer centres of Thiruvallur, Kanchipuram and Villupuram. Distance from the centre may not have significant impact on readmissions.

Respiratory distress, chest pain, haemoptysis and extrapulmonary symptoms like joint pain and swelling, headache, dizziness and psychiatric symptoms are common complications for which readmissions were seen.
Apart from this adverse drug reaction is the main reason for patients to get readmitted in these populations. Common and frequent side effects of GI tolerance, arthralgia, arthritis, dizziness and psychiatric illnesses were seen in these patients.

\section{DISCUSSION}

The reason behind this evaluation is to assess if there was any possibility of improving the final outcome and to prevent the economic burden if patients are getting readmitted for preventable reasons.

Total number of patients admitted under MDR TB ward during the assessment period is 790 and those who were readmitted were 419 , which are approximately more than $50 \%$. Mean duration of stay in these patients is about 20 days. It is an immense economic burden on the family and state.

Taylor et $\mathrm{al}^{6}$ in their study suggest similar pattern in cause of admissions. But they studied all causes of hospital admissions in tuberculosis patients, wherein they reported initial admissions account for almost more than three quarters of the total admissions. Their study also included MDR TB and HIV population too which are minimum numbers in theirs compared to drug sensitive $\mathrm{Tb}$ patients. Even though our study concentrates mainly on MDR TB, the pattern of readmissions are similar here too.

Readmissions were significantly more common in the males and more particularly in the 20 - 50 age groups, which is obviously the most productive group for the country and the family. Now, this may be seen in 2 different perspectives. The working group might have work stress and emotional stress that they would not have got enough physical and emotional rest they need during the course of MDR TB treatment. This might lead to decrease in physical capacity and recurrent readmissions.

At every juncture throughout their admissions in this centre, there was every effort made to make these patients understand the necessity of nutrition, emotional and physical stability for the chemotherapy to work. They would undergo repeated counselling by staff nurse in charge, medical officers as well as a social counsellor. In spite of these maximal efforts, patient were needed by their family to provide for their daily bread and butter.

When patients were asked about their personal history, most of them revealed alcoholism. Alcoholism as such can be asserted to be a cause of malnutrition, but it also plays a huge factor in default and drug malabsorption. This significantly increases the probability of MDR TB readmissions as well as it may lead to more morbid and mortal XDR TB. These patients when probed deeply also gave history of substance abuse, but of minimum numbers only. Substance abuse and alcoholism may cause a direct reason for the patient to get admitted. But this factor is more significant than the population without alcoholism and substance abuse for readmissions is beyond the scope of this study.

With every patient, these patients were also counselled and they received psychiatric consultations and medications.

Common complications for which the patients were admitted were exacerbation of respiratory symptoms, chest pain, haemoptysis and extrapulmonary symptoms like joint pain and swelling, headache, dizziness and psychiatric symptoms. These symptoms cannot be managed by 
peripheral hospitals, but they need an expert panel multidisciplinary physicians to manage.

Common adverse effects were GI intolerance, peripheral neuropathy, arthritis, change in visual disturbances, psychiatric episodes, etc. These patients were readmitted, evaluated completely and then stabilised and sent back to their respective peripheral centres for continuation of the treatment.

Patients having pneumothorax were around 14. In these 14 , some had hydropneumothorax too. They were treated symptomatically, some with conservative management and others intervened by ICD. In the ward side ICD, care was given properly with antibiotic and oxygen support. About 11 patients developed bronchopleural fistula, wherein treatment was conservative. None of these patients necessitated surgical intervention according to the records available.

Anti-tb treatment is toxic even with the first line, but worse is the second line Tb drugs. One of the common cause of default is long course of treatment and toxicity of the drugs. Out of 419 readmissions, about 41 were due to GI intolerance and about 20 were due to drug induced hepatitis. These patients were managed symptomatically and supported by intravenous fluid support.

About 23 of the 419 readmissions were due to arthralgia. They were managed by topical analgesics and interestingly their uric acid levels were normal. About 21 patients had dermatitis and were managed by topical and oral antihistamines.

Comorbidities include anaemia $(\mathrm{N}=95)$, cor pulmonale, diabetes mellitus $(\mathrm{N}=134)$, systemic hypertension $(\mathrm{N}=31)$ and coronary artery disease apart from retroviral disease that is already discussed. These disorders were diagnosed after admissions, treated for by specialists at each step. Anaemia was mostly nutritional corrected by nutritional vitamin supplements and sometimes patients had undisclosed haemorrhoids, which were revealed after deeper probing and examinations. Some of these patients needed blood transfusions. Some patients were newly diagnosed with diabetes, as they were in a chronic low intake state the random blood sugar were normal. On further examination by glucose challenge did they revealed diabetic status. Systemic hypertension and coronary artery disease were also diagnosed only during admissions. In these patients, dyspnoea was related to the uncontrolled hypertension or coronary disease, which the primary physician could have easily missed thinking of airway and ventilation disorders due to damage of lung parenchyma. These patients were treated by specific specialists and managed accordingly.

The implications of this study may be used further to study the population deeply as multidrug as well as extensively drug resistant tuberculosis are on the rise and there needs to be a system in place to tackle this problem of complications, adverse effects of drugs or other social reasons. The first and foremost might be to prevent the occurrence of these diseases by identifying $\mathrm{Tb}$ in early stages and treating them effectively. If there is a possibility of identifying drug resistance early this could be undertaken and initiation of treatment at the earliest and counselling patients and their family members itself can reduce some of the social reasons for readmissions.
Even though these patients are counselled repeatedly and family members are made aware of the situations, the system on its part has to play a role. Repeated counselling by the DOTS provider and any abnormal reactions on patient that is noticed by the DOTS provider should be brought to the notice of the concerned physician, so that early intervention may settle some of these issues like vitamin supplements, antihistamine administration, topical analgesic administration and antiemetic and anti-ulcer agents.

Physicians need more orientation programs to identify and treat these complications and adverse effects early. They need to counsel these patients whenever they visit their outpatient department also. The efficacy of these interventions are hard to tell and beyond the scope of this study.

Even though most of the readmissions were just like those in drug sensitive group, some of the adverse effects like psychiatric disturbances and cor pulmonale are more in the drug resistant $\mathrm{Tb}$ treatment settings.

\section{CONCLUSION}

Adverse drug reaction (34.7\%), Default (12.5\%) and Complications of MDR TB (16.6\%) are common causes for readmission.

Social causes have significant proportion of readmission (9\%).

Readmissions are more common in patients with HIV and substance abuse.

Adverse drug reactions and complications are manageable only by an expert panel. Orientation courses for medical fraternity in peripheries will help to sort this out.

Default may be due to several reasons. Foremost may be due to alcoholism and social issues, which might be prevented by simple but efficient counselling sessions.

\section{REFERENCES}

[1] Chan ED, Laurel V, Strand MJ, et al. Treatment and outcome analysis of 205 patients with multidrugresistant tuberculosis. Am J Respir Crit Care Med 2004;169(10):1103-9.

[2] Phillips PP, Gillespie SH, Boeree M, et al. Innovative trial designs are practical solutions for improving the treatment of tuberculosis. Journal of Infectious Diseases 2012;205(Suppl 2):S250-7.

[3] Eker B, Ortmann J, Migliori GB, et al. Multidrug- and extensively drug-resistant tuberculosis, Germany. Emerg Infect Dis 2008;14(11):1700-6.

[4] Fischl MA, Daikos GL, Uttamchandani RB, et al. Clinical presentation and outcome of patients with hiv infection and tuberculosis caused by multiple-drugresistant bacilli. Ann Intern Med 1992;117(3):184-90.

[5] Bhagwat S, Pujari V, Rajurkar S, et al. Hospital admissions for tuberculosis in an Indian metropolitan city. International Journal of Science and Research (IJSR) 2014;3(8):283-5.

[6] Taylor Z, Marks SM, Burrows RNM, et al. Causes and costs of hospitalization of TB patients in the United States. Int J Tuberc Lung Dis 2000;4(10):931-9. 